\title{
An X\#Ray Variable Millisecond Pulsar in the Globular Cluster 47 Tucanae: Closing the Link to Low\#Mass X\#Ray Binaries
}

\section{Citation}

Bogdanov, Slavko, Jonathan E. Grindlay, and Maureen van den Berg. 2005. “An X\#Ray Variable Millisecond Pulsar in the Globular Cluster 47 Tucanae: Closing the Link to Low\#Mass X\#Ray Binaries." The Astrophysical Journal 630 (2): 1029-36. https://doi.org/10.1086/432249.

\section{Permanent link}

http://nrs.harvard.edu/urn-3:HUL.InstRepos:41399913

\section{Terms of Use}

This article was downloaded from Harvard University's DASH repository, and is made available under the terms and conditions applicable to Other Posted Material, as set forth at http:// nrs.harvard.edu/urn-3:HUL.InstRepos:dash.current.terms-of-use\#LAA

\section{Share Your Story}

The Harvard community has made this article openly available. Please share how this access benefits you. Submit a story.

Accessibility 


\title{
AN X-RAY VARIABLE MILLISECOND PULSAR IN THE GLOBULAR CLUSTER 47 TUCANAE: CLOSING THE LINK TO LOW-MASS X-RAY BINARIES
}

\author{
Slavko Bogdanov, Jonathan E. Grindlay, and Maureen van den Berg \\ Harvard-Smithsonian Center for Astrophysics, 60 Garden Street, Cambridge, MA 02138; \\ sbogdanov@cfa.harvard.edu, josh@cfa.harvard.edu,mvandenberg@cfa.harvard.edu \\ Received 2005 February 7; accepted 2005 May 25
}

\begin{abstract}
We report the discovery of peculiar X-ray spectral variability in the binary radio millisecond pulsar PSR J0024-7204W in the globular cluster 47 Tuc. The observed emission consists of a dominant nonthermal component, which is eclipsed for a portion of the orbit, and a thermal component, which appears to be persistent. We propose that the nonthermal X-rays originate in a relativistic intrabinary shock, formed due to interaction between the relativistic particle wind from the pulsar and matter from the main-sequence companion star, while the thermal photons are from the heated magnetic polar caps of the millisecond pulsar. At optical wavelengths, the emission exhibits large-amplitude variations at the orbital period, which can be attributed to heating of one side of the tidally locked secondary star by the pulsar wind. The observed X-ray and optical properties of PSR J0024-7204W are remarkably similar to those of the low-mass X-ray binary and X-ray millisecond pulsar SAX J1808.4-3658 in quiescence. This supports the conjecture that the nonthermal X-ray emission and optical modulations seen in the SAX J1808.4-3658 system in a quiescent state are due to interaction between the wind from a reactivated rotationpowered pulsar and matter from the companion star. The striking similarities between the two systems provide support for the long-sought connection between millisecond radio pulsars and accreting neutron star systems.
\end{abstract}

Subject headings: pulsars: general — pulsars: individual (PSR J0024-7204W) — stars: neutron — X-rays: stars

Online material: color figures

\section{INTRODUCTION}

Radio millisecond pulsars (MSPs) form a separate class of rotation-powered pulsars, characterized by small spin periods, $P \lesssim 25 \mathrm{~ms}$, and spin-down rates, $\dot{P}=d P / d t \sim 10^{-19}$ to $10^{-21}$, implying surface magnetic field strengths of $B \propto(P \dot{P})^{1 / 2} \sim$ $10^{8}-10^{9} \mathrm{G}$ and characteristic ages of $\tau \equiv P / 2 \dot{P} \gtrsim 10^{9}$ yr. These neutron stars (NSs) are widely believed to be the end-products of a more exotic channel of binary evolution, involving an extended period of accretion of matter and angular momentum from a close stellar companion in X-ray binary systems (Alpar et al. 1982; Bhattacharya \& van den Heuvel 1991). After a period of $10^{7}-10^{8} \mathrm{yr}$, they acquire millisecond spin periods and after accretion ceases are reactivated as radio pulsars. This theory is supported by the fact that over $\sim 75 \%$ of the known MSPs in the Galactic disk are found in binary systems, whereas this is the case for only $\sim 1 \%$ of the general pulsar population. ${ }^{1}$ The strongest support so far for the connection between MSPs and $\mathrm{X}$-ray binaries comes from the discovery of coherent millisecond X-ray pulsations in the low-mass X-ray binary (LMXB) transient SAX J1808.4-3658 (Wijnands \& van der Klis 1998) and subsequently five ${ }^{2}$ other systems (see Campana et al. 1998 for a review). However, a firm connection between the two classes of objects is yet to be established as no LMXB has been detected as a radio pulsar and no MSP has shown X-ray properties of a quiescent LMXB (qLMXB).

The globular cluster 47 Tuc (NGC 104) is known to host at least 22 radio millisecond pulsars (Camilo et al. 2000; Freire

\footnotetext{
${ }^{1}$ Australia Telescope National Facility Pulsar Catalog, available at http:// www.atnf.csiro.au/research/pulsar/psrcat.

${ }^{2}$ For the discovery of the latest X-ray millisecond pulsar, see http:// www.astronomerstelegram.org/?read $=353$.
}

et al. 2001, 2003; Camilo \& Rasio 2005 and references therein). Many of these MSPs, however, have flux densities below the sensitivity of the Parkes radio telescope used to detect them and are only observable on rare occasions when strong interstellar scintillation focusing effects bring them above the detection limit. Thus, to date, 17 of the 22 MSPs have been detected sufficiently frequently to allow a precise determination of their positions from radio-timing solutions. During the only radio observation in which PSR J0024-7204W (henceforth 47 Tuc W or MSP W) was detected, ${ }^{3}$ this $2.35 \mathrm{~ms}$ pulsar was found to be in a $3.2 \mathrm{hr}$ binary orbit and to undergo eclipses, but its position could not be determined accurately (Camilo et al. 2000). Fortunately, Hubble Space Telescope (HST) observations of 47 Tuc have been used to match the binary period and phase of the modulations of a variable star with that of MSP W and thus determine the position of the MSP with subarcsecond precision (Edmonds et al. 2002). Surprisingly, the optical counterpart of 47 Tuc $\mathrm{W}$ was found to be a $\gtrsim 0.13 M_{\odot}$ main-sequence star, which is unexpected for a MSP binary companion since in all but one other case the secondary star is greatly evolved. This intriguing result implies that either the companion is the star that very recently finished spinning up MSP W or that it is not the original secondary star but was instead exchanged for the lowmass $\left(<0.1 M_{\odot}\right)$ companion, which, in the process, was liberated from the binary. The HST identification showed MSP W to be positionally coincident with the X-ray source W29, first detected by a 72 ks Chandra X-Ray Observatory ACIS-I observation of the core of 47 Tuc (Grindlay et al. 2001). The X-ray spectrum was found to be relatively hard, suggestive of nonthermal emission,

\footnotetext{
3 After submission of this article for review, it was brought to our attention that this MSP has been detected again (P. Freire 2005, private communication).
} 
atypical of the MSP sample in 47 Tuc, but similar to PSR J1740-5340 in the globular cluster NGC 6397 (D'Amico et al. 2001; Grindlay et al. 2002), the only other known MSP with a nondegenerate stellar companion.

\section{OBSERVATIONS AND DATA ANALYSIS}

We have employed the superb spatial resolution of Chandra and HST to further investigate the unusual properties of the 47 Tuc W system. The X-ray data set consists of four additional 65 ks observations of the core of 47 Tuc performed with the Chandra ACIS-S instrument. These deep observations have resulted in the detection of $\sim 300$ sources within the half-mass radius of 47 Tuc (Heinke et al. 2005), including the X-ray counterparts for each of the MSPs with known positions (S. Bogdanov et al. 2005, in preparation). The optical HST data consist of three sets of archival observations acquired with the High Resolution Camera (HRC) on the Advanced Camera for Surveys (ACS) and one set of observations performed contemporaneously with the Chandra observations using the Wide Field Camera (WFC) on the ACS. Table 1 summarizes all X-ray and optical observations used in this analysis.

The initial data reduction and image processing of the X-ray observations were performed using the $\mathrm{CIAO}^{4}$ version 3.0 software package and are discussed in detail elsewhere (Heinke et al. 2005). For the spectral and variability analyses, the Chandra observations were first filtered to include only those events with energies between 0.3 and $8.0 \mathrm{keV}$. Subsequently, the X-ray photons from MSP W were obtained from a circular region with a $1^{\prime \prime}$ radius around the position of the optical counterpart, as this circle encloses $>90 \%$ of the total energy for an assumed $0.5-$ $1.0 \mathrm{keV}$ thermal source. The net count rate was then obtained by subtracting a background taken from three source-free regions on the image. To facilitate the spectral analysis, the $\sim 300$ net photons were grouped into energy bins, while requiring that each bin contain at least 15 counts. For all spectral fits the hydrogen column density toward 47 Tuc was fixed at $N_{\mathrm{H}}=(1.3 \pm 0.3) \times$ $10^{20} \mathrm{~cm}^{-2}$ (Gratton et al. 2003; Cardelli et al. 1989; Predehl \& Schmitt 1995). An X-ray light curve was obtained by folding the observations at the binary period using the latest ephemeris obtained from radio pulse timing observations (P. Freire et al. 2005, unpublished).

The optical colors and variability of 47 Tuc W were first investigated in our ACS WFC data. Unfortunately, due to the large pixel scale of the WFC $\left(0.049\right.$ pixel $\left.^{-1}\right)$ and the presence of several relatively bright stars around the position of 47 Tuc W, the faint optical source was not detected in the individual ACS observations. Since the images were dithered relative to each other with subpixel offsets, we could create combined images in each filter with improved spatial resolution using the Space Telescope Science Institute multidrizzle routines. In these phase-averaged images, the companion of 47 Tuc W is visible near the limit of detection. However, the large errors in the magnitude $(\sim 0.3-0.5 \mathrm{mag})$ prevented us from obtaining reliable light curves or a significant limit on the $\mathrm{H} \alpha$ emission expected from this system. Thus, we have used the higher resolution $\left(0\right.$ ".027 pixel $\left.^{-1}\right)$ ACS/HRC archival data, where 47 Tuc $\mathrm{W}$ is clearly visible when at maximum brightness. We measured magnitudes using aperture photometry on the distortion-corrected pipeline-processed images. A small aperture (2 pixel radius) was chosen to minimize contamination by the flux of nearby

\footnotetext{
${ }^{4}$ Chandra Interactive Analysis of Observations, available at http://asc .harvard.edu/ciao.
}

TABLE 1

Summary of the X-Ray (Chandra) and Optical (HST) Observations Used in This Analysis

\begin{tabular}{|c|c|c|}
\hline Telescope/Instrument & Epoch of Observation & Observation ID \\
\hline Chandra/ACIS-S. & 2002 Sep 29 & 2735 \\
\hline Chandra/ACIS-S. & 2002 Sep 30 & 2736 \\
\hline Chandra/ACIS-S. & 2002 Oct 2 & 2737 \\
\hline Chandra/ACIS-S ................ & 2002 Oct 11 & 2738 \\
\hline HST/ACS-HRC .... & 2002 Apr 5 & $9028^{\mathrm{a}}$ \\
\hline 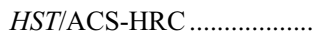 & 2002 Apr $12-14$ & 9019 \\
\hline HST/ACS-HRC .... & 2002 Jul 24 & 9443 \\
\hline 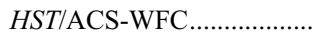 & 2002 Sep $30-$ Oct $11^{\mathrm{b}}$ & 9281 \\
\hline
\end{tabular}

a See also Edmonds et al. (2002).

b Simultaneous with the Chandra ACIS-S observations.

stars. For a few frames, mostly for those in which the counterpart of 47 Tuc W was near minimum brightness, a small change in the choice of background regions resulted in magnitude differences of up to $0.8 \mathrm{mag}$. We corrected these magnitudes to an aperture of 6 pixels using aperture photometry of several bright and relatively isolated stars within 200 pixels of the optical counterpart of 47 Tuc W. For each filter, we checked the variation of this correction as a function of time but found no indication for a systematic trend. This led us to apply a time-averaged aperture correction per filter. Next, we used the filter-dependent encircled energy curves from Sirianni et al. (2005), ${ }^{5}$ which give the fraction of total source counts as a function of aperture radius, to correct the magnitudes to an infinite aperture (5".5; see Sirianni et al. 2005 for details). We deemed as unreliable those measurements for which these differences would result in flux differences of more than $\sim 30 \%$ and removed them from our data set. The remaining data points do not show a systematic difference when compared to magnitudes derived for a different choice of background. Instrumental magnitudes were converted to magnitudes in the STmag system using the most recent filter-dependent sensitivities. ${ }^{6} \mathrm{We}$ used these fluxes to obtain sinusoidal fits to the light curves and make phase-dependent spectral energy distributions (SEDs). The times of midobservation were converted to photometric phases using the existing radio ephemeris (P. Freire et al. 2005, unpublished).

\section{THE X-RAY SPECTRUM}

The X-ray spectrum of 47 Tuc $\mathrm{W}$ in the $0.3-8.0 \mathrm{keV}$ range suggests that the emission consists of at least two components: a dominant nonthermal (power-law) and a fainter thermal component. The latter is fit equally well by either a simple blackbody (BB) or a NS hydrogen atmosphere (NSA) model (Romani 1987; Zavlin et al. 1996; Lloyd 2003). After excluding the photons between orbital phases $\phi=0.4-0.7$ to correct for the bias in the best-fit parameters introduced by the variability of the nonthermal component (see $\S 4$ and Fig. 1), we obtain the following best-fit models. If we assume a BB model for the thermal component, we find that the spectrum can be described by a nonthermal component with a power-law photon index of $\Gamma=1.13 \pm 0.35$ and $\mathrm{a} \mathrm{BB}$ with an effective temperature of $T_{\text {eff }}=(1.56 \pm 0.28) \times$ $10^{6} \mathrm{~K}$ and an effective emitting radius of $R_{\text {eff }}=0.45 \pm 0.43 \mathrm{~km}$ $\left(\chi_{\nu}^{2}=1.22\right.$ for 14 degrees of freedom), with a total unabsorbed

\footnotetext{
5 Available at http://acs.pha.jhu.edu/instrument/calibration/photometry.

${ }^{6}$ See http://www.stsci.edu/hst/acs/analysis/zeropoints.
} 


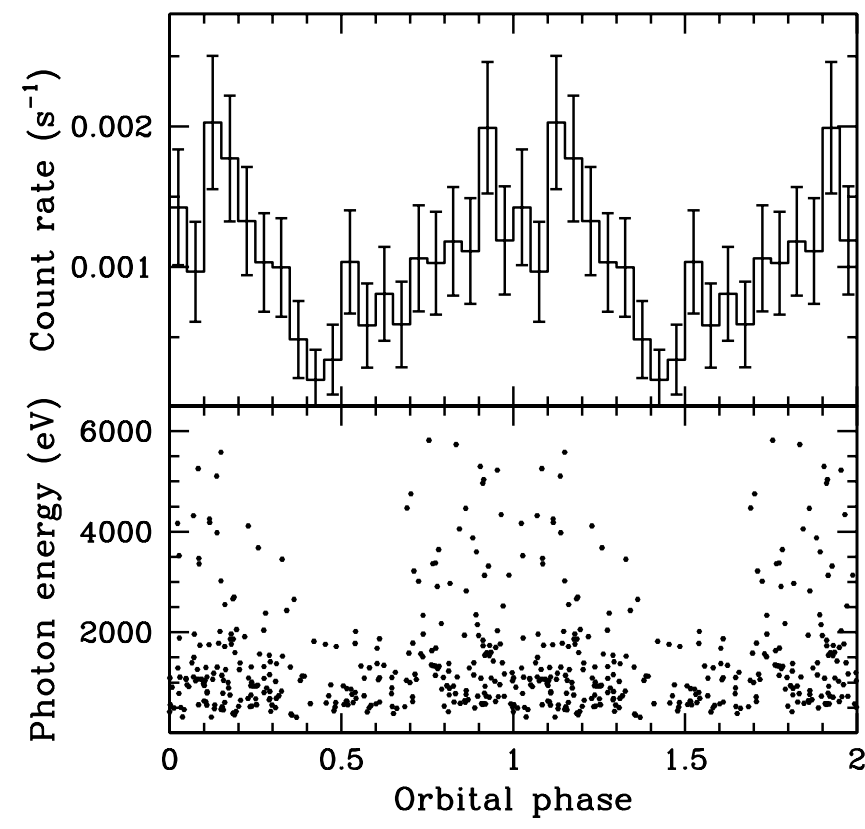

FIG. 1.-Top: X-ray light curve of PSR J0024-7204W in the $0.3-6.0 \mathrm{keV}$ band, folded over its binary period. Note the X-ray minimum at $\phi \approx 0.45$ instead of $\phi=0.5$, the position of the optical minimum. The uncertainty in the absolute phase is $>10^{-4}$. Bottom: Energy of each photon detected within $1^{\prime \prime}$ of the optical position of 47 Tuc W vs. orbital phase. The data shown in this plot cover an integer number (21) of binary orbits to ensure uniform phase coverage. Note the remarkable absence of photons with energies above $2 \mathrm{keV}$ and the reduction in the number of soft photons between orbital phases 0.4 and 0.7 . Two orbital cycles are shown for clarity.

flux of $F_{\mathrm{X}}=(1.4 \pm 0.3) \times 10^{-14} \mathrm{ergs} \mathrm{cm}^{-2} \mathrm{~s}^{-1}$. On the other hand, if we consider a NSA model and assume a gravitational redshift of $z_{g}=0.31$ at the NS surface, we obtain $\Gamma=1.14 \pm$ $0.35, T_{\text {eff }}=0.95_{-0.24}^{+0.31} \times 10^{6} \mathrm{~K}, R_{\text {eff }}=0-2.3 \mathrm{~km}\left(\chi_{\nu}^{2}=1.17\right.$ for 14 degrees of freedom), and a total unabsorbed flux of $F_{\mathrm{X}}=$ $(1.5 \pm 0.3) \times 10^{-14} \mathrm{ergs} \mathrm{cm}^{-2} \mathrm{~s}^{-1}$. In both cases, the nonthermal component contributes with $\sim 75 \%$ of the total flux. For a distance to 47 Tuc of $4.85 \mathrm{kpc}$ (Gratton et al. 2003) we obtain a total X-ray luminosity of $L_{\mathrm{X}}=(4 \pm 1) \times 10^{31} \mathrm{ergs} \mathrm{s}^{-1}$ and a nonthermal luminosity of $L_{\mathrm{X}, \mathrm{NT}} \approx(2-3) \times 10^{31} \mathrm{ergs} \mathrm{s}^{-1}(0.3-8.0 \mathrm{keV})$. All errors quoted above represent $1 \sigma$ uncertainties in the derived parameters. We note that although an $F$-test does not indicate that a composite model is statistically preferred over a pure power-law model (but with a steeper spectral index of $\Gamma=1.7 \pm 0.1$ ), the $\mathrm{X}$-ray light curve, discussed in $\S 4$, suggests that the thermal component in the spectrum is genuine. In addition, the best-fit values for $T_{\text {eff }}$ and $R_{\text {eff }}$ are quite similar to what is observed in MSPs with predominantly thermal spectra, such as the majority of MSPs in 47 Tuc (Grindlay et al. 2002; S. Bogdanov et al. 2005 , in preparation). The marginally acceptable fits suggest that a second thermal component is probably present in the spectrum, as seen in the nearby MSPs J0437-4715 and J0030+0451 (Zavlin et al. 2002; Becker \& Achenbach 2002).

Thermal emission from MSPs is believed to originate from the magnetic polar caps of the underlying NS, heated by a backflow of energetic particles from the pulsar magnetosphere (see, e.g., Harding \& Muslimov 2002; Zhang \& Chang 2003 and references therein). On the other hand, nonthermal emission from MSPs can be produced by two different mechanisms. In the most luminous MSPs, B1821-24 and B1937+21 (Becker \& Trümper 1999 ), the narrow X-ray pulse profiles indicate that the nonthermal emission is beamed and must originate in the pulsar magnetosphere, near the light cylinder, and is probably synchro- tron and curvature radiation from charged particles accelerated to relativistic speeds in the strong magnetic field. Alternatively, nonthermal radiation can be produced by the interaction of the pulsar wind with the binary companion or the interstellar medium. The resulting shock wave is expected to be a prominent source of high-energy photons, via the synchrotron or inverse Compton radiation produced by the highly accelerated particles at the shock front and in the flow downstream from the shock (Arons \& Tavani 1993).

\section{X-RAY VARIABILITY}

In the 47 Tuc $\mathrm{W}$ system, we do not know a priori the exact nature of the observed nonthermal emission. Although the Chandra ACIS-S observations preclude pulsation analysis due to the limited time resolution $(3.2 \mathrm{~s})$, the longer timescale variability contains a wealth of information about the origin of the nonthermal X-rays. In particular, the light curve folded at the binary period exhibits dramatic variations as a function of orbital position (Fig. 1, upper panel). Using Poisson statistics, we find that the observed variability is significant at the $99.9 \%$ confidence level.

Surprisingly, a comparison of the X-ray colors from different orbital phases shows that the spectrum of MSP W softens significantly around the minimum for $\sim 30 \%$ of the orbit, with no counts detected above $2 \mathrm{keV}$ (see Fig. 1, lower panel). In addition, at phases between 0.4 and 0.7 , we find a decline of about $70 \%$ in the number of soft photons $(<2 \mathrm{keV})$. The remaining $\sim 30 \%$ are consistent with being entirely due to the underlying thermal (BB or NSA) component, although some of these photons may be due to partial eclipses of the nonthermal source. In any case, there is strong indication that the nonthermal component is occulted for roughly one third of the orbit, most likely by the main-sequence secondary star. Note that the underlying $\mathrm{X}$-ray source cannot be eclipsed by diffuse material within or around the binary, as this would be manifested primarily by absorption of soft photons.

The characteristics of the X-ray spectrum and light curve are suggestive of emission originating from two different locations within the binary. As stated above, the thermal component most likely originates from the heated polar caps of the MSP. On the other hand, the observed X-ray eclipses are inconsistent with a magnetospheric origin of the nonthermal photons. If these $\mathrm{X}$-rays did originate from the pulsar magnetosphere, one would expect both emission components to undergo much shorter (at most, $\sim 10 \%$ of the orbit) total eclipses, characterized by very rapid ingress and egress and centered at exactly $\phi=0.5$. The observed eclipses also suggest a localized nonthermal source, rather than an extended circumbinary X-ray emission region that could form, for instance, by interaction of the pulsar wind with the interstellar medium.

The behavior of the hard X-ray emission can be naturally explained by the presence of a relativistic shock wave within the binary, produced by interaction between the pulsar wind and matter from the secondary star. This shock forms at some distance from the MSP, where the ram pressure of the pulsar wind balances the pressure exerted by the gas from the companion star. The length of the X-ray eclipse ( $\sim 30 \%$ of the orbit) suggests that this occurs much closer to the companion star than the MSP. The shock can then be eclipsed by the secondary for a substantial portion of the orbit, even if the MSP is never occulted. As noted above, if the MSP is occulted by the secondary, which would occur for $80^{\circ} \lesssim i \leq 90^{\circ}$, the eclipse should last for no more than $2 \%-5 \%$ of the orbit. Unfortunately, the limited number of photons observed at the 


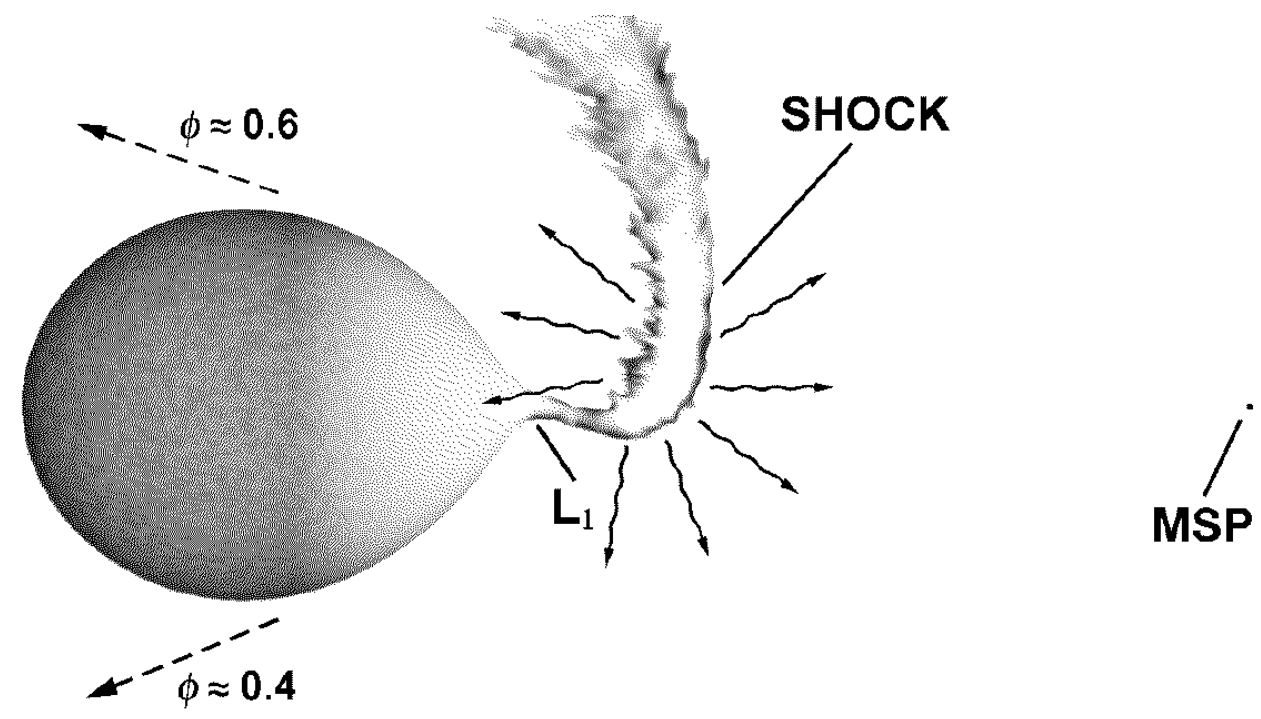

FIG. 2.- Representation of the 47 Tuc W binary illustrating the key features of the system for the case of a Roche lobe filling companion, for assumed masses of 1.4 and $0.15 M_{\odot}$ for the MSP and main-sequence companion, respectively. Here the orbit is depicted face-on $\left(i=0^{\circ}\right)$ to better show the geometry of the X-ray-emitting shocked region. The sense of rotation of the system is counterclockwise and is in the plane of the page. The dashed arrows point in the general direction of the observer roughly before (bottom) and after (top) the X-ray minimum. [See the electronic edition of the Journal for a color version of this figure.]

X-ray minimum (only three detected counts in a bin of width 10 minutes) prevents us from determining whether this is indeed the case and thus limiting the maximum allowed binary inclination.

If the secondary star is underfilling its Roche lobe (RL), the observed shock could result from the pulsar wind impinging on and evaporating material off of the surface of the companion or interacting with the stellar wind, generated due to the corotation forced by tidal coupling of the orbital motion to the deep convection layers of the secondary star (see, e.g., Wijers \& Paczyński 1993 and references therein). More likely, the main-sequence companion may be overflowing its RL, as a result of bloating due to irradiation by the energetic particle wind from the MSP (see, e.g., Podsiadlowski 1991; D’Antona \& Ergma 1993; Vilhu et al. 1994). The shock can then be produced by the interaction of the pulsar wind with a stream of gas from the companion issuing through the inner Lagrange point (L1). If this is indeed the case, it allows us to place a conservative lower limit on the orbital inclination by noting that at $\phi \approx 0.45$, the nonthermal $\mathrm{X}$-ray source is completely occulted. For the RL geometry of a 1.4 $M_{\odot}$ MSP and a $0.13-0.29 M_{\odot}$ companion, corresponding to the allowed range of inclinations $90^{\circ} \geq i \gtrsim 27^{\circ}$ (Edmonds et al. 2002), we find $i \gtrsim 35^{\circ}$.

An interesting feature of the X-ray eclipse is the apparent sharp increase in the count rate immediately after the X-ray minimum. If real, this emission may be the signature of Doppler boosting of the particle flow downstream from the shock as it travels around the secondary star (Arons \& Tavani 1993). This effect can also account for the possible drop in the count rate at $\phi \approx 1$, corresponding to the portion of the orbit at which the particles in the shock are accelerated primarily in the direction away from the observer. Another notable property of the eclipse is its asymmetry, characterized by a rapid ingress and slower egress. In addition, the minimum in the X-ray flux occurs at $\phi=$ $0.4-0.45$, i.e., before the optical minimum at superior conjunction $(\phi=0.5)$. This suggests the presence of a swept-back shocked region that is elongated perpendicular to and is asymmetric about the semimajor axis of the binary. Such a geometry arises because, as the gas from the secondary interacts with the pulsar wind, it can no longer corotate along with the secondary star and must therefore fall behind. Figure 2 illustrates the likely appearance of the nonthermal emission region for the RL overflow case as inferred from the X-ray light curve. The geometry of the shock is similar if the companion star is contained within its RL, except that for the case of evaporative mass loss the shocked "cometary" tail would originate at or very near the surface of the star. We note that a wind from the secondary star could also result in mass outflow through L1 and a configuration similar to that shown in Figure 2. In all cases, when viewed at $\phi \approx 0.4$, the apparent length of the shocked region is relatively small, explaining the short duration of ingress. Conversely, at $\phi \approx 0.6$ the projected length of the emission region is larger, causing a gradual transition out of eclipse. The relative durations of ingress and egress, as well as the length of the eclipse, permit us to roughly constrain the geometry of the gas stream and the shock. Taking $i=90^{\circ}$, and a shock approximately at L1, we find an upper limit on the length of the shocked stream of $l \lesssim 2 \times 10^{10} \mathrm{~cm}$, comparable to the radius of the companion, and a distance of $d \lesssim 5 \times 10^{9} \mathrm{~cm}$, from the surface of the companion to the shock.

\section{LARGE-AMPLITUDE OPTICAL VARIABILITY}

Figure 3 shows the HST ACS/HRC light curves in the F435W, F475W, F555W, and F606W bands. For these filters there is sufficient phase coverage to fit the amplitude and average flux level of variation. For the fit we assumed a sinusoidal light curve and the latest radio ephemeris (P. Freire et al. 2005, unpublished). To create SEDs, the fitted fluxes at maximum, mean, and minimum brightness were first corrected for the extinction toward 47 Tuc using the filter-dependent conversions in Table 32 of Sirianni et al. (2005) for the spectral type (G2) closest to that of the counterpart of MSP W (see below for the results of our blackbody fits). Since the detailed spectrum of the counterpart is unknown, it is difficult to estimate the corresponding systematic errors. From comparison with the only two conversions provided for other spectral types (O5 and $\mathrm{M} 0$ ), we expect that these are small. The effective wavelengths that are used in the SEDs are derived with the task calcphot in 

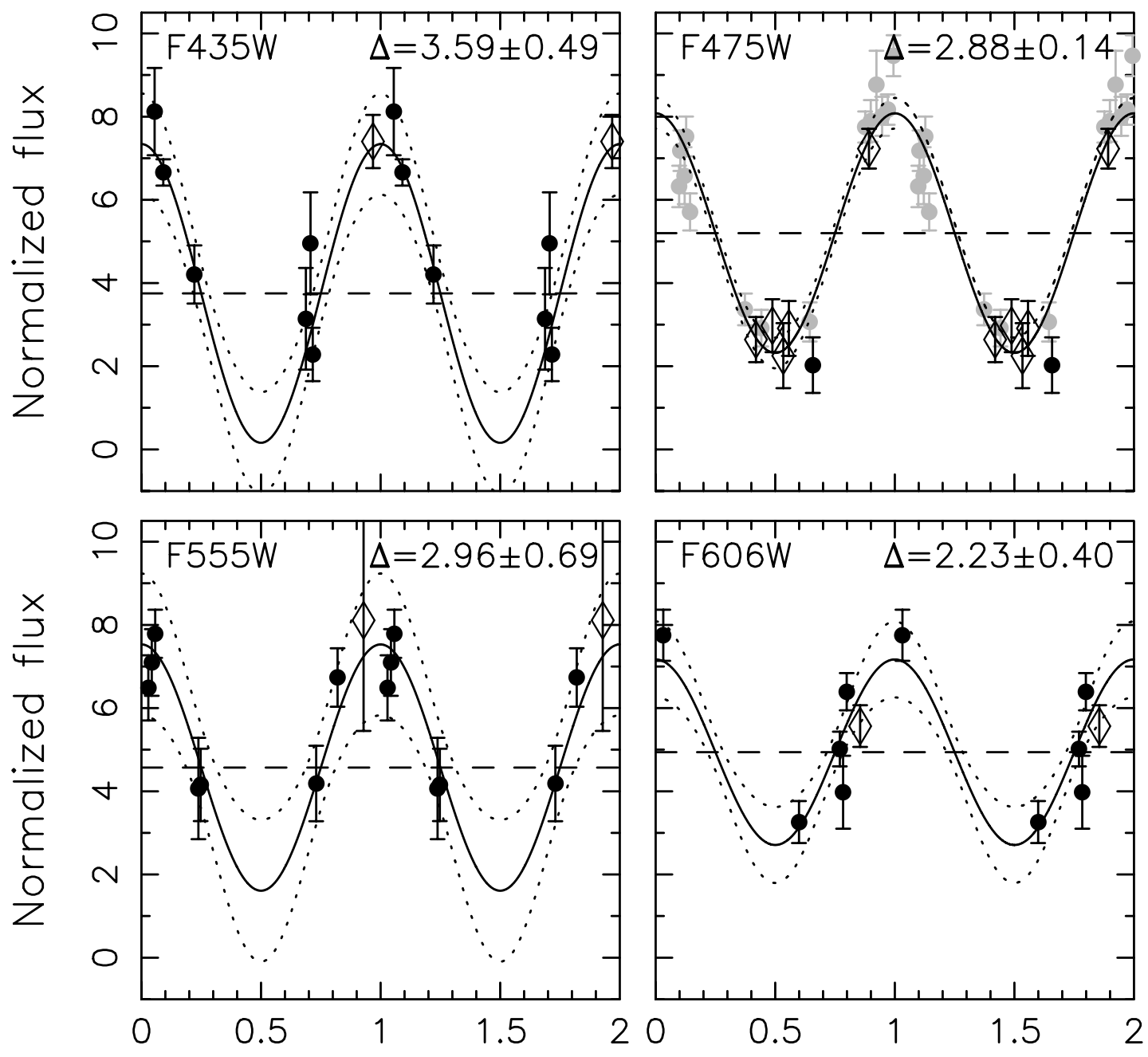

\section{Photometric phase Photometric phase}

FIG. 3.- HST HRC light curves of the optical counterpart of $47 \mathrm{Tuc} \mathrm{W}$ in different filters folded at the photometric period. The flux is in units of $10^{-18} \mathrm{ergs} \mathrm{s}^{-1} \mathrm{~cm}^{-2} \AA^{-1}$. The data from observing programs 9019,9028 , and 9443 are plotted with solid circles, gray circles, and open diamonds, respectively. For each filter the solid line represents the sine curve with the best-fit amplitude and average flux level, with period and phasing from the most recent radio timing ephemeris (P. Freire et al. 2005, unpublished). The dotted lines represent $2 \sigma$ error margins, while the dashed lines give the average flux levels. The $H S T$ filter and $\Delta$, the amplitude of variation $( \pm 1 \sigma)$, are given in the upper left corner of each panel. Note the trend of decreasing amplitude with increasing wavelength. Two orbital cycles are shown for clarity.

IRAF STSDAS $^{7}$ from folding the spectrum of a reddened $[E(B-V)=0.023] 5500 \mathrm{~K}$ blackbody with the filter response curves. The resulting SEDs for photometric phases $0,0.25 / 0.75$, and 0.5 were then fit to a BB spectrum with fixed $R_{\text {eff }}$ but variable $T_{\text {eff. }}$.

We find that for the SED at maximum brightness, the data are best fit with $T_{\text {eff }}$ between $\sim 6800 \mathrm{~K}$, for $R_{\text {eff }}=0.15 R_{\odot}$ (the radius appropriate for an unheated $0.13 M_{\odot}$ star) and $\sim 5400 \mathrm{~K}$ for $R_{\mathrm{eff}}=0.26 R_{\odot}$ (corresponding to the $\mathrm{RL}$ radius for $i=90^{\circ}$ and $\left.M_{\mathrm{MSP}}=1.4 M_{\odot}\right)$. Similarly, the fit to the SED at mean brightness results in a range of $T_{\text {eff }} \sim 6200-5000 \mathrm{~K}$, while at minimum brightness we obtain $T_{\text {eff }} \sim 5200-4400 \mathrm{~K}$ for the same range of stellar radii. We note that although the fits are consistent with $R_{\mathrm{eff}}<R_{\mathrm{RL}}$, this does not necessarily mean that the companion is not RL filling. It is more likely that only a portion

\footnotetext{
${ }^{7}$ IRAF is distributed by the National Optical Astronomy Observatories, which are operated by the Association of Universities for Research in Astronomy, Inc., under cooperative agreement with the National Science Foundation.
}

of the hemisphere facing the MSP is actually heated due to less efficient heating near the poles (see Fig. 2). Furthermore, small apparent values for $R_{\text {eff }}$ can result from a viewing angle effect for $i<90^{\circ}$. Given the uncertainties in the inclination and the details of particle heating, we can only constrain the temperature difference between the heated and unheated areas of the secondary to $\Delta T_{c} \sim 1000-2400 \mathrm{~K}$, under the assumption that $R_{\text {eff }}$ at optical maximum is less than or equal to $R_{\text {eff }}$ at minimum.

An extrapolation of the nonthermal X-ray emission to optical wavelengths shows that the nonthermal emission produced in the intrabinary shock contributes negligibly to the optical emission, even if we take into account the large uncertainties in the absolute magnitudes of the optical data and the nonthermal X-ray photon index (see Fig. 4). Therefore, the observed largeamplitude variability must be due to a temperature difference of the two hemispheres of the companion, which can be attributed to the heating of one side of the tidally locked secondary star by the MSP, and not from the shock. This scenario is consistent with the light curves in Figure 3, which show a trend 


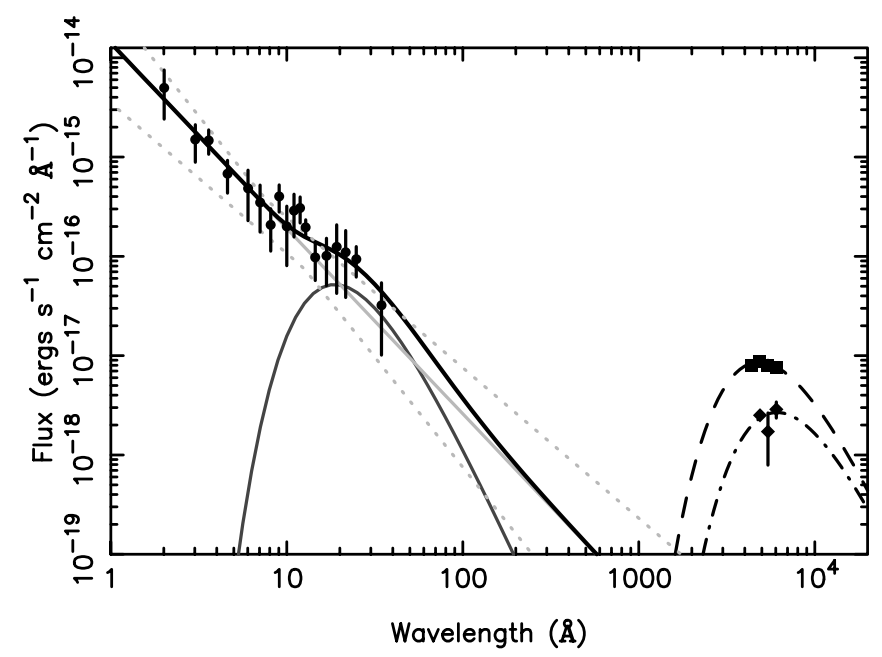

FIG. 4.-Broadband spectrum of 47 Tuc W showing the X-ray data (circles) and optical data at maximum (squares) and minimum (diamonds). Also shown is the best-fit model for the total emission (thick black line), as well as for the individual emission components: thermal emission from the MSP (dark gray line), blackbody emission from the secondary star at optical maximum and minimum (dashed and dot-dashed lines, respectively) assuming $R_{\mathrm{eff}}=0.20 R_{\odot}$, and nonthermal emission from the intrabinary shock (light gray line). The dotted lines delineate the bounds of the $1 \sigma$ uncertainty in the best-fit nonthermal (power-law) model. [See the electronic edition of the Journal for a color version of this figure.]

of decreasing amplitude of the optical modulation with increasing wavelength. As we discuss below, both the overall X-ray to optical spectrum and variability are very similar to those observed in the SAX J1808.4-3658 system (Campana et al. 2002, 2004).

The combined X-ray and optical data permit us to determine the origin of the energy supplied to the secondary star. In particular, we find that the total X-ray and UV flux from the intrabinary shock is insufficient to heat the face of the companion star to the observed temperature $\left(T_{H} \sim 5400-6800 \mathrm{~K}\right)$. Therefore, we conclude that the heating of the secondary must instead be due to the relativistic particle wind of the MSP, generated at the expense of the rotational energy of the underlying NS. The rate of this energy loss is given by $\dot{E}=4 \pi^{2} I \dot{P} / P^{3}$, where $I$ is the NS moment of inertia, typically assumed to be $10^{45} \mathrm{~g} \mathrm{~cm}^{2}$. Unfortunately, 47 Tuc $\mathrm{W}$ is rarely detected at radio frequencies, which does not allow a determination of the pulsar spin-down rate $\dot{P}$. Hence, we have no direct measure of $\dot{E}$. Nonetheless, we can use the correlation between the observed thermal X-ray luminosity $\left(L_{\mathrm{X}, T}\right)$ and $\dot{E}$ found for the 47 Tuc MSPs to obtain a crude estimate of this parameter. Using $L_{\mathrm{X}, T} \sim(0.5-1) \times 10^{31} \mathrm{ergs} \mathrm{s}^{-1}$ and the empirical relation $\log L_{\mathrm{X}, T}=0.59 \log \dot{E}+10.0$ (Grindlay et al. 2002), we obtain $\dot{E} \sim 1 \times 10^{35} \mathrm{ergs} \mathrm{s}^{-1}$. Assuming an isotropic pulsar wind, we find that the total incident power on the secondary star is $L_{\text {irr }} \sim 1 \times 10^{33} \mathrm{ergs} \mathrm{s}^{-1}$. This value is sufficient to heat the face of the companion star to the observed luminosity $\left(L_{H} \sim 1 \times 10^{32} \mathrm{ergs} \mathrm{s}^{-1}\right)$, if we allow for a re-radiation efficiency of order 0.1 . Furthermore, the flux from the MSP wind at the surface of the secondary $\left(\sim 10^{12} \mathrm{ergs} \mathrm{cm}^{-2} \mathrm{~s}^{-1}\right)$ is conducive to bloating of the star to a significantly larger radius (see Fig. 1 in Podsiadlowski 1991), provided that the particles constituting the wind deposit their energy below the stellar photosphere. Given that the pulsar wind is probably composed of highly relativistic electrons, positrons, and ions, which are able to penetrate deep into the stellar interior, this condition for expansion of the star can be satisfied, implying that the secondary star is most likely
RL filling. Finally, the flux from the pulsar wind at L1 is consistent with the observed value of $L_{\mathrm{X}, \mathrm{NT}} \approx 3 \times 10^{31} \mathrm{ergs} \mathrm{s}^{-1}$, if we take into account the particle acceleration efficiency $\epsilon_{a} \simeq 0.2$ (Arons \& Tavani 1993), as well as the fraction of the wind interacting with matter from the companion $\left(\sim 10^{-3}\right)$.

\section{EMISSION PROPERTIES OF THE INTRABINARY SHOCK}

The compact nature of the 47 Tuc W binary ensures that the intrabinary shock is located in a relatively strong pulsar magnetic field. Therefore, we expect synchrotron emission to be the principal energy loss mechanism in the shock. However, the proximity of the shock wave to the secondary star implies that the relativistic electrons in the shock are immersed in a "sea" of optical photons emanating from the secondary star. Thus, inverse Compton scattering (ICS) may also be an important production mechanism for high-energy photons.

The magnetic field strength immediately upstream of the shock is given by $B_{1}=[\sigma /(1+\sigma)]^{1 / 2}\left(\dot{E} / c f_{p} d^{2}\right)^{1 / 2}$, where $\sigma$ is the ratio of the magnetic energy flux to the kinetic energy flux, $f_{p}$ is the geometric factor that defines the fraction of the sky into which the pulsar wind is emitted, and $d$ is the distance between the MSP and the shock (Arons \& Tavani 1993). For simplicity, we take $d \approx 6.4 \times 10^{10} \mathrm{~cm}$, corresponding approximately to the distance from the MSP to L1 assuming $M_{\mathrm{MSP}}=1.4 M_{\odot}$ and $i=$ $60^{\circ}$. For an isotropic pulsar wind $\left(f_{p}=1\right)$, we obtain $B_{1} \approx 2 \mathrm{G}$ and $B_{1} \approx 30 \mathrm{G}$ corresponding to the two possible cases of a kinetic energy dominated ( $\sigma=0.003$ as in the case of the Crab Nebula) and a magnetically dominated $(\sigma \gg 1)$ wind, respectively. This implies a magnetic field strength beyond the shock of $B_{2}=3 B_{1} \sim 6 \mathrm{G}$ or $B_{2} \sim 90 \mathrm{G}$, respectively.

Production of the observed $\epsilon=0.3-8 \mathrm{keV}$ photons via synchrotron therefore requires relativistic electrons with Lorentz factors $\gamma=2.4 \times 10^{5}\left(\epsilon / B_{2}\right)^{1 / 2} \sim\left(1 \times 10^{4}\right)-\left(5 \times 10^{5}\right)$. From these values, we obtain a radiative loss time of $t_{\text {synch }}=$ $5.1 \times 10^{8}\left(\gamma B_{2}^{2}\right)^{-1} \sim 1-60 \mathrm{~s}$ (Rybicki \& Lightman 1979). If we consider a power-law distribution of electron energies, i.e., $n_{e}(\gamma) \propto \gamma^{-p}$, where $p$ is related to the photon index by $p=$ $2 \Gamma+1$ (Rybicki \& Lightman 1979), and assume a roughly cylindrical shocked emission region with length $l \sim 2 \times 10^{10} \mathrm{~cm}$ and radius $\sim 2 \times 10^{9} \mathrm{~cm}$, we find that the required electron density is $n_{e} \sim 10^{7}$ or $n_{e} \sim 10^{4} \mathrm{~cm}^{-3}$, corresponding to $\sigma=$ 0.003 and $\sigma \gg 1$, respectively. These values imply energy densities in electrons of $U_{e} \sim 10^{2}-\left(5 \times 10^{3}\right)$ or $U_{e} \sim 10^{5}-(5 \times$ $\left.10^{6}\right) \mathrm{ergs} \mathrm{cm}^{-3}$. The value for $\sigma \gg 1$ is comparable to the magnetic energy density $U_{B}=B_{2}^{2} / 8 \pi \sim 1-300 \mathrm{ergs}^{-3}$. Thus, based on equipartition of energy arguments, the wind is most likely magnetically dominated.

We note that if ICS were to dominate the X-ray emission, the radiating electrons need to be only weakly accelerated to $\gamma \sim$ 100 , implying radiative loss times of $t_{\mathrm{ICS}}=\left(U_{B} / U_{\mathrm{ph}}\right) t_{\mathrm{synch}} \sim$ 10-100 hr, where $U_{\mathrm{ph}}=R_{c} \sigma_{B} T_{c}^{4} /\left(R_{*} c\right) \sim 1 \mathrm{ergs} \mathrm{\textrm {cm } ^ { - 3 }}$ is the seed photon energy density, while $R_{c}, T_{H}$, and $R_{*}$ are the radius, temperature, and distance from the surface of the secondary star, respectively. The implied $U_{e} \sim 10^{7} \mathrm{ergs} \mathrm{cm}^{-3}$, however, greatly exceeds the values of $U_{B}$ and $U_{\mathrm{ph}}$. Therefore, ICS is an unlikely soft X-ray production mechanism in 47 Tuc W.

Future detailed multiwavelength observations of the 47 Tuc W system may reveal more information concerning the nature of the shock emission, which, in turn, may provide insight into the little understood properties of MSP winds, collisionless relativistic shocks, and particle acceleration mechanisms. The existence of a regularly eclipsed shock makes 47 Tuc W well suited for studies of these phenomena. 


\section{DISCUSSION}

\subsection{Comparison with Other MSPs}

The data presented here have revealed that 47 Tuc $\mathrm{W}$ exhibits $\mathrm{X}$-ray variability that is unique among the MSPs (as is evident in Fig. 1). These variations can be most easily explained by the presence of a relativistic shock within the binary that is regularly eclipsed by the secondary star. An intrabinary shock is also believed to be present in PSR B1957+20, the so-called black widow eclipsing pulsar (Fruchter et al. 1988). This MSP has a nonthermal spectrum with $\Gamma=1.9 \pm 0.5$ and $L_{\mathrm{X}}=2.7 \times 10^{31} \mathrm{ergs} \mathrm{s}^{-1}$ (0.5-7.0 keV; Stappers et al. 2003), for an assumed distance of $2.5 \mathrm{kpc}$ (Cordes \& Lazio 2002). These values are comparable to those for MSP W and provide further support for the presence of a shock in the 47 Tuc W system. However, we emphasize that 47 Tuc W is a fundamentally different binary than PSR B1957+20 and similar eclipsing systems, such as 47 Tuc J, O, and R (S. Bogdanov et al. 2005, in preparation), which contain much less massive $\left(\sim 0.03 M_{\odot}\right)$, greatly evolved secondary stars.

MSP W possesses many characteristics similar to PSR J1740-5340 (J1740), the only known MSP in the globular cluster NGC 6397 (D'Amico et al. 2001; Ferraro et al. 2003; Sabbi et al. 2003). Specifically, J1740 exhibits radio eclipses, $\mathrm{X}$-ray emission that is seemingly hard and possibly variable (Grindlay et al. 2002) and is bound to a $\sim 0.3 M_{\odot}$ "red straggler" companion that is currently filling its Roche lobe. In addition, the shape of the $\mathrm{H} \alpha$ line observed in this $32.5 \mathrm{hr}$ binary system indicates the presence of a swept-back stream of gas protruding from the companion, as the one possibly present in 47 Tuc W (see Fig. 2). However, unlike 47 Tuc W, J1740 shows no signature of wind-driven heating of the companion in the photometric light curve (Kaluzny et al. 2003; Orosz \& van Kerkwijk 2003). This finding is consistent with the recently revised value of $\dot{E} \approx 3.3 \times 10^{34} \mathrm{ergs} \mathrm{s}^{-1}$ (Bassa \& Stappers 2004), which indicates that the flux from the pulsar wind incident on the $\sim 1.6 R_{\odot}$ companion is too small to have a measurable effect. Therefore, in this system RL overflow is not the result of bloating by irradiation from the MSP but rather a consequence of evolution off of the main sequence. Perhaps the most peculiar feature of the J1740 binary is the presence of $\mathrm{He}$ I absorption localized in a thin longitudinal strip near the surface of the secondary (Ferraro et al. 2003). The shape of such a heated region could be due to irradiation of the companion by a highly anisotropic pulsar wind, preferentially emitted in the orbital plane. However, this would require the MSP spin and orbital angular momentum vectors to be exactly aligned. The thin strip can be more plausibly explained by the existence of an equatorial wind, emanating from the tidally locked companion star due to its forced rapid corotation, that is interacting with the pulsar wind. Alternatively, the $\mathrm{He}$ I absorption could originate in the swept-back tail of material, as the one shown in Figure 2 for 47 Tuc W.

\subsection{The MSP-LMXB Connection}

The unusual X-ray and optical properties of 47 Tuc W can serve toward finally establishing the long-suspected connection between LMXBs and MSPs. This is now possible because the 47 Tuc W system appears to be more typical of a qLMXB system than a MSP binary system, as it contains a main-sequence companion that may be RL filling. In addition, the X-ray spectrum of 47 Tuc W system exhibits remarkable similarities to that of the LMXB transient SAX J1808.4-3658 (hereafter J1808) in quiescence. $X M M$-Newton observations of the latter system have revealed that during the long periods between outbursts
TABLE 2

A Comparison of the Observed Properties of 47 Tuc W and J1808

\begin{tabular}{|c|c|c|}
\hline Parameter & PSR J0024-7204W & SAX J1808.4-3658 \\
\hline$L_{\mathrm{X}}\left(\operatorname{ergs~s}^{-1}\right) \ldots \ldots \ldots \ldots$ & $3 \times 10^{31}(0.3-8 \mathrm{keV})$ & $5 \times 10^{31}(0.5-10 \mathrm{keV})$ \\
\hline 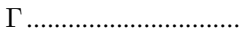 & $1.13 \pm 0.35(1 \sigma)$ & $1.4_{-0.3}^{+0.6}(3 \sigma)$ \\
\hline$\dot{E}\left(\operatorname{ergs~s}^{-1}\right) \ldots \ldots \ldots \ldots \ldots$ & $\sim 10^{35 \mathrm{a}}$ & $?$ \\
\hline$L_{i}\left(\operatorname{ergs~s}^{-1}\right) \ldots \ldots \ldots \ldots$ & $\sim 1 \times 10^{31}$ & $\lesssim 3 \times 10^{31}$ \\
\hline$L_{\text {irr }}\left(\operatorname{ergs~} \mathrm{s}^{-1}\right) \ldots \ldots \ldots \ldots$ & $\sim 4 \times 10^{33}$ & $\gtrsim 4 \times 10^{33}$ \\
\hline$T_{H}(\mathrm{~K}) \ldots \ldots \ldots \ldots \ldots \ldots$ & $5400-6800$ & $6200_{-380}^{+850 \mathrm{~b}}$ \\
\hline$L_{H}\left(\operatorname{ergs~s}^{-1}\right) \ldots \ldots \ldots \ldots$ & $\sim 1 \times 10^{32}$ & $\sim 4 \times 10^{31}$ \\
\hline$\Delta T_{c}(\mathrm{~K}) \ldots \ldots \ldots \ldots \ldots \ldots$ & $1000-2400$ & $\sim 1000$ \\
\hline
\end{tabular}

Notes. $-L_{i}$ is the estimated intrinsic bolometric luminosity of a $0.15 M$ secondary star in the absence of heating from the pulsar, while $T_{H}$ and $L_{H}$ are the temperature and bolometric luminosity of the heated face of the companion. The other parameters listed are defined in the text.

a Estimate.

b S. Campana (2004, private communication).

( $\sim 2 \mathrm{yr}$ ), this $2.01 \mathrm{hr}$ binary has an X-ray spectrum that is seemingly purely nonthermal, with $\Gamma=1.4_{-0.3}^{+0.6}$ and an X-ray luminosity of $L_{\mathrm{X}}=5 \times 10^{31} \mathrm{ergs} \mathrm{s}^{-1}$ in the $0.5-10 \mathrm{keV}$ band (Campana et al. 2002). Moreover, recent optical observations of the bloated $\sim 0.05 M_{\odot}$ brown dwarf companion of J1808 show sinusoidal variations at the orbital period (Campana et al. 2004), implying a temperature difference of $\Delta T_{c} \sim 1000 \pm 300 \mathrm{~K}$ between the two faces of the companion, quite similar to that reported here for 47 Tuc W. Finally, the observed nonthermal emission in J1808 also cannot account for the optical flux as well as irradiating luminosity $\left(L_{\text {irr }} \gtrsim 4 \times 10^{33} \mathrm{ergs} \mathrm{s}^{-1}\right)$ required to produce the observed heating of the secondary star (see Fig. 1 of Campana et al. 2004). The X-ray and optical properties of 47 Tuc $\mathrm{W}$ and $\mathrm{J} 1808$ are compared in Table 2.

The primary difference between $\mathrm{J} 1808$ in quiescence and 47 Tuc $\mathrm{W}$ is the lack of radio pulsations from the former. One possibility is that the radio beams are not favorably oriented, rendering J1808 undetectable at radio wavelengths. Alternatively, the radio signals from the nascent MSP expected in J1808 could be obscured by a diffuse circumbinary envelope of material, formed by the gas flowing out from the companion that is ultimately expelled from the system by the pulsar wind. Such an envelope is likely present around the 47 Tuc W system as well and may explain, in part, why this MSP is occulted for $\sim 40 \%$ of the orbit and is rarely detected at radio frequencies (Freire 2005). If this is the case, it would be yet another common feature of the two systems.

We propose that the great similarities in the values of the power-law index and the nonthermal X-ray luminosity derived for 47 Tuc W and J1808 point to a common origin of the nonthermal X-ray emission in both systems. Also, it is highly probable that the source of energy supplied to the heated companion in both systems is the same as well. ${ }^{8}$ If this is indeed the case, then, as originally suggested by Burderi et al. (2003) and Campana et al. (2004), in the J1808 system there exists a pulsar wind, powered by the rotational energy of the NS, which is constraining the outflow of material from the companion and preventing its accretion onto the NS. As in the 47 Tuc W system, the presence of this relativistic particle wind is manifested via the nonthermal X-ray emission produced by interaction of this wind with matter flowing out from the companion, as well as

\footnotetext{
${ }^{8}$ For J1808, unlike the case of 47 Tuc W, no simultaneous X-ray and optical data are available, and the best limit on $L_{\mathrm{X}}$ is $<10^{34} \mathrm{ergs} \mathrm{s}^{-1}$, as measured 2 days before the optical observations.
} 
through the optical variability caused by heating of one side of the tidally locked companion. By analogy, this would exclude an accretion disk (e.g., Garcia et al. 2001 and references therein) or a propeller mechanism as the source of hard X-rays in J1808 (Wijnands 2003 and references therein), since we know that in 47 Tuc $\mathrm{W}$ accretion is inhibited by the pulsar wind. The remarkable similarities between 47 Tuc $\mathrm{W}$ and J1808 provide strong support for the claim that in episodes of quiescence, the NS in the qLMXB system $\mathrm{J} 1808$ has already been reactivated as a rotation-powered millisecond pulsar. This, in turn, gives indirect evidence in favor of the long-standing hypothesis that LMXBs are indeed progenitors of MSPs.

\subsection{X-Ray Source Populations}

The implications of the 47 Tuc W system on studies of X-ray source populations are profound since low-luminosity $\left(L_{\mathrm{X}} \sim\right.$ $3 \times 10^{31}$ ergs s $^{-1}$ ) hard X-ray sources, which would otherwise be classified as cataclysmic variables $(\mathrm{CVs})$, i.e., accreting white dwarf systems (Grindlay et al. 2001), may, instead, be MSP binaries in some cases. The same holds true for the lowluminosity, hard-spectrum qLMXBs categorized as black hole (BH) systems (Garcia et al. 2001; Rutledge et al. 2000) based on the absence of thermal emission of a cooling NS. The optical heating effects of the pulsar wind, as seen in 47 Tuc W, would allow MSPs to be discovered and distinguished from CVs or $\mathrm{BH}$ qLMXBs since the low X-ray luminosities and the absence of an energetic wind do not result in significant heating of the secondary star for comparably short orbital periods. We also note that the discriminant of X-ray to optical flux ratios, $F_{\mathrm{X}} / F_{V}$, which might be used to distinguish qLMXBs from CVs or MSPs, is not useful for these transition qLMXB-MSP systems: J1808 and MSP W have $\log \left(F_{\mathrm{X}} / F_{V}\right) \sim 0.7$ and 0.4 , respectively, which are typical values for CVs or qLMXBs but much larger than a "typical" MSP with an evolved companion. For comparison, the corresponding value for MSP U in 47 Tuc, whose companion is likely a helium white dwarf (Edmonds et al. 2001), is $\log \left(F_{\mathrm{X}} / F_{V}\right) \sim-1.15$ (Heinke et al. 2005).

We thank Craig Heinke for both his original analysis of the deep Chandra observations and many fruitful discussions, Peter Edmonds for discussions of his initial 47 Tuc W discovery analysis using archival HST data, and Paulo Freire for providing the most up-to-date binary ephemeris for MSP W. We are also grateful to Fernando Camilo and Sergio Campana for insightful discussions, Don Lloyd for use of his neutron star atmosphere models, and Rob Hynes, whose BinSim software package was used to generate parts of Figure 2. This work was supported in part by NASA Chandra grant GO2-3059A and HST grants HSTGO-0944 and HST-GO-10009. The research presented here has made use of the NASA Astrophysics Data System.

Facilities: CXO(ACIS), HST(ACS)
Alpar, M. A., Cheng, A. F., M. A. Ruderman, M. A., \& Shaham, J. 1982, Nature, 300, 728

Arons, J., \& Tavani, M. 1993, ApJ, 403, 249

Bassa, C. G., \& Stappers, B. W. 2004, A\&A, 425, 1143

Becker, W., \& Achenbach, B. 2002, in Proc. 270th WE-Heraeus Seminar on Neutron Stars, Pulsars, and Supernova Remnants, ed. W. Becker, H. Lech, \& J. Trümper (MPE Rep. 278; Garching: MPE), 64

Becker, W., \& Trümper, J. 1999, A\&A, 341, 803

Bhattacharya, D., \& van den Heuvel, E. P. J. 1991, Phys. Rep., 203, 1

Burderi, L., Di Salvo, T., D’Antona, F., Robba, N. R., \& Testa, V. 2003, A\&A, 404, L43

Camilo, F., Lorimer, D. R., Freire, P., Lyne, A. G., \& Manchester, R. N. 2000, ApJ, 535, 975

Camilo, F., \& Rasio, F. A. 2005, in ASP Conf. Ser. 328, Binary Radio Pulsars, ed. F. A. Rasio \& I. H. Stairs (San Francisco: ASP), 147

Campana, S., Colpi, M., Mereghetti, S., Stella, L., \& Tavani, M. 1998, A\&A Rev., 8, 279

Campana, S., et al. 2002, ApJ, 575, L15

. 2004, ApJ, 614, L49

Cardelli, J. A., Clayton, G. C., \& Mathis, J. S. 1989, ApJ, 345, 245

Cordes, J. M., \& Lazio, T. J. W. 2002, preprint (astro-ph/0207156)

D’Amico, N., Lyne, A. G., Manchester, R. N., Possenti, A., \& Camilo, F. 2001, ApJ, 548, L171

D’Antona, F., \& Ergma, E. 1993, A\&A, 269, 219

Edmonds, P. D., Gilliland, R. L., Camilo, F., Heinke, C. O., \& Grindlay, J. E. 2002, ApJ, 579, 741

Edmonds, P. D., Gilliland, R. L., Heinke, C. O. Grindlay, J. E., \& Camilo, F. 2001, ApJ, 557, L57

Ferraro, F. R., Sabbi, E., Gratton, R., Possenti, A., D’Amico, N., Bragaglia, A., \& Camilo, F. 2003, ApJ, 584, L13

Freire, P. C. C. 2005 , in ASP Conf. Ser. 328, Binary Radio Pulsars, ed. F. A. Rasio \& I. H. Stairs (San Francisco: ASP), 405

Freire, P. C., Camilo, F., Kramer, M., Lorimer, D. R., Lyne, A. G., Manchester, R. N., \& D' Amico, N. 2003, MNRAS, 340, 1359

Freire, P. C., Camilo, F., Lorimer, D. R., Lyne, A. G., Manchester, R. N., \& D'Amico, N. 2001, MNRAS, 326, 901
Fruchter, A. S., Stinebring, D. R., \& Taylor, J. H. 1988, Nature, 333, 237

Garcia, M. R., McClintock, J. E., Narayan, R., Callanan, P., Barret, D., \& Murray, S. S. 2001, ApJ, 553, L47

Gratton, R. G., Bragaglia, A., Carretta, E., Clementini, G., Desidera, S., Grundahl, F., \& Lucatello, S. 2003, A\&A, 408, 529

Grindlay, J. E., Camilo, F., Heinke, C. O., Edmonds, P. D., Cohn, H., \& Lugger, P. 2002, ApJ, 581, 470

Grindlay, J. E., Heinke, C., Edmonds, P. D., \& Murray, S. S. 2001, Science, 292, 2290

Harding, A. K., \& Muslimov, A. G. 2002, ApJ, 568, 862

Heinke, C. O., Grindlay, J. E., Edmonds, P. D., Cohn, H. N., Lugger, P. M., Camilo, F., Bogdanov, S., \& Freire, P. C. 2005, ApJ, 625, 796

Kaluzny, J., Rucinski, S. M., \& Thompson, I. B. 2003, AJ, 125, 1546

Lloyd, D. A. 2003, preprint (astro-ph/030356)

Orosz, J. A., \& van Kerkwijk, M. H. 2003, A\&A, 397, 237

Podsiadlowski, Ph. 1991, Nature, 350, 136

Predehl, P., \& Schmitt, J. H. M. M. 1995, A\&A, 293, 889

Romani, R. W. 1987, ApJ, 313, 718

Rutledge, R. E., Bildsten, L., Brown, E. F., Pavlov, G. G., \& Zavlin, V. E. 2000, ApJ, 529, 985

Rybicki, G. B., \& Lightman, A. P. 1979, Radiative Processes in Astrophysics (New York: Wiley)

Sabbi, E., Gratton, R., Ferraro, F. R., Bragaglia, A., Possenti, A., D’Amico, N., \& Camilo, F. 2003, ApJ, 589, L41

Sirianni, M., et al. 2005, PASP, submitted

Stappers, B. W., Gaensler, B. M., Kaspi, V. M., van der Klis, M., \& Lewin,

W. H. G. 2003, Science, 299, 1372

Vilhu, O., Ergma, E., \& Fedorova, A. 1994, A\&A, 291, 842

Wijers, R. A. M. J., \& Paczyński, B. 1993, ApJ, 415, L115

Wijnands, R. 2003, ApJ, 588, 425

Wijnands, R., \& van der Klis, M. 1998, Nature, 394, 344

Zavlin, V. E., Pavlov, G. G., Sanwal, D., Manchester, R. N., Trümper, J., Halpern, J. P., \& Becker, W. 2002, ApJ, 569, 894

Zavlin, V. E., Pavlov, G. G., \& Shibanov, Yu. A. 1996, A\&A, 315, 141

Zhang, L., \& Chang, K. S. 2003, A\&A, 398, 639 